\title{
Kinetics and Determining Factors of the Virologic Response to Antiretrovirals during Pregnancy
}

\author{
Adriana Weinberg, ${ }^{1}$ Jeri E. F. Harwood, ${ }^{1}$ Elizabeth J. McFarland, ${ }^{1}$ Jennifer Pappas, ${ }^{2}$ \\ Jill Davies, ${ }^{3}$ Kay Kinzie, ${ }^{1}$ Emily Barr, ${ }^{1}$ Suzanne Paul, ${ }^{1}$ Carol Salbenblatt, ${ }^{2}$ Elizabeth Soda, ${ }^{4}$ \\ Anna Vazquez, ${ }^{1}$ Charles A. Peloquin, ${ }^{5}$ and Myron J. Levin ${ }^{1}$
}

\author{
${ }^{1}$ Department of Pediatrics, University of Colorado Denver School of Medicine, Aurora, CO 80045, USA \\ ${ }^{2}$ Department of Pediatrics, The Children's Hospital, Aurora, CO 80045, USA \\ ${ }^{3}$ Department of Obstetrics and Gynecology, University of Colorado Denver School of Medicine, Aurora, CO 80045, USA \\ ${ }^{4}$ University of Colorado Denver School of Medicine, Aurora, CO 80045, USA \\ ${ }^{5}$ Department of Infectious Diseases, National Jewish Hospital, Denver, CO 80206, USA
}

Correspondence should be addressed to Adriana Weinberg, adriana.weinberg@ucdenver.edu

Received 23 June 2009; Accepted 15 October 2009

Recommended by Grace John-Stewart

\begin{abstract}
HIV-infected pregnant women with undetectable plasma HIV RNA concentrations at delivery pose a minimal risk of vertical transmission. We studied the kinetics and the determinants of the virologic response to antiretroviral therapy in 117 consecutive pregnancies. Patients who initiated therapy during pregnancy had a VL decrease of 2 and $2.5 \log _{10}$ after 4 and 24 weeks, respectively. Therapeutic drug monitoring (TDM) of the protease inhibitors administered in doses recommended for nonpregnant adults resulted in below-target concentrations in $29 \%, 35 \%$, and $44 \%$ of 1st, 2nd, and 3rd trimester measurements, respectively, but low drug concentrations did not correlate with virologic failure. Demographic characteristics, antiretroviral experience prior to pregnancy, baseline VL, or use of specific antiretrovirals did not affect the virologic response. Adherence to $\geq 95 \%$ of prescribed doses and utilization of psychosocial services were associated with undetectable plasma HIV RNA at delivery. In conclusion, the virologic responses of pregnant and nonpregnant adults share similar charactersitics.
\end{abstract}

Copyright (C) 2009 Adriana Weinberg et al. This is an open access article distributed under the Creative Commons Attribution License, which permits unrestricted use, distribution, and reproduction in any medium, provided the original work is properly cited.

\section{Introduction}

Highly active antiretroviral (ARV) therapy (HAART) has decreased HIV mother-to-child transmission (MTCT) to $<2 \%$ in the US and other countries where ARVs are readily available $[1-5]$. This was achieved by suppressing maternal HIV replication. In addition, scheduled Cesarean section decreases the risk of MTCT $[1,6,7]$. Recent studies, however, suggest that Cesarean sections are not of significant benefit when plasma HIV RNA is undetectable at delivery $[2,3]$. Since Cesarean sections increase the cost of deliveries and risk of maternal complications, understanding the kinetics of the virologic response to HAART during pregnancy may avoid performing unnecessary Cesarean sections.

The dominant risk factor for MTCT is high maternal viral load at delivery [8]. In nonpregnant individuals, HAART generally decreases HIV plasma RNA and increases
CD4+ cells in parallel. This relationship has prompted the use of $\mathrm{CD} 4+$ counts as a surrogate measure of response to therapy when plasma HIV RNA measurements are not readily available. In pregnancy, however, this approach may not be valid, as pregnancy is associated with lower CD4+ numbers. The kinetics of plasma HIV RNA and CD4+ cells in response to HAART during pregnancy have been reported in separate studies $[9,10]$. The information provided by these studies suggested that there may be a disconnect between changes in CD4+ cells and plasma HIV RNA during pregnancy. In this study, we analyzed these two parameters in parallel.

Another important issue for prevention of MTCT (PMTCT) is optimization of drug regimens during pregnancy, which typically include 2 nucleoside reverse transcriptase inhibitors (NRTI) and a protease inhibitor (PI). Pharmacokinetics and therapeutic drug monitoring (TDM) 
studies during pregnancy showed that absorption and distribution of PIs differ in 3rd trimester pregnant women compared with nonpregnant adults, such that dose adjustments may be warranted [11-13]. However, there is a lack of information regarding PI pharmacokinetics during early pregnancy.

To address these issues, we conducted a retrospective chart review of pregnancies managed by the Children's Human Immunodeficiency Program (CHIP) in Denver. In August 1997, CHIP became the reference center for the care of HIV-infected pregnant women in Colorado and neighboring states. These women come from a relatively broad patient population that is highly representative of the HIV epidemic in the Southwestern US.

\section{Patients and Methods}

2.1. CHIP PMTCT Program. Patients were cared for by a multidisciplinary team, comprising specialists in adult and pediatric infectious diseases, obstetrics and maternalfetal medicine, nursing, social work, mental health, and nutrition. Basic HIV-specific treatment consisted of $\geq 3$ ARV including $\geq 2$ classes of ARV. HIV genotype was determined before treatment initiation in patients with plasma HIV RNA $\geq 1000$ copies/mL. Adherence was facilitated by distributing pill boxes, watches, and pagers, office counseling or home visits, and direct observed therapy. Plasma HIV RNA and CD4+ cells were measured at 2-6 week intervals.

PI plasma concentrations were assessed using highpressure liquid chromatography assays performed at the National Jewish Hospital pharmacology laboratory, certified by the AIDS Clinical Trials Group and compliant with the Clinical Laboratory Improvements Amendments. For TDM, drugs were administered in clinic with or without food, as indicated. Trough concentrations were obtained immediately before the dose. Peak concentrations of lopinavir (LPV) were measured 4 hours after administration of LPV/ritonavir (LPVr) and of other PIs 2 hours after administration of the drug.

Treatment was modified based on drug levels, virologic response, safety, and tolerability. Centralized treatment decisions were made by the physician in charge of the maternal care of the PMTCT program in consultation with the primary infectious diseases provider of the patient, if different from the PMTCT physician.

The HIV-specific obstetrical plan included intravenous zidovudine (AZT) administration during delivery except for patients on stavudine, who continued their oral medications. Cesarean sections were scheduled at 38 weeks of gestation for consenting patients with VL $>1000$ copies $/ \mathrm{mL}$ at $\geq 34$ weeks gestation, as indicated by other medical obstetrical conditions or patients' choice.

Infants were treated during the first 6 weeks of life with AZT. When the maternal VL at the last visit prior to delivery was $\geq 5,000$ copies $/ \mathrm{mL}$ or $>1000$ copies $/ \mathrm{mL}$ with vaginal delivery, the neonate received 2 additional drugs from 2 ARV classes for the first 4 weeks of life.
2.2. Chart Review. This study was reviewed by the local IRB and deemed exempt from IRB approval due to the retrospective nature of the study and absence of disclosure of any personal information. Pregnancies with delivery between Aug. 1997 (inception of the CHIP PMTCT program) and Dec. 2005 (initiation of chart review) were identified in the CHIP database. Information was abstracted for pregnancies with $\geq 16$-week duration and $\geq 2$ visits at CHIP, using studyspecific forms.

2.3. Statistical Analysis. Analyses assumed a two-sided test of hypothesis with a 0.05 significance level. Associations between categorical variables were determined using Fisher's exact test. Wilcoxon rank-sum test was used for comparisons of continuous variables between two groups. Changes in VL and CD4+ T-cell counts were tested using a signed-rank test.

To determine the kinetics of the virologic response, $\log _{10}$ VL was modeled as smooth functions of time on treatment using a natural cubic B-spline transformation on time [14]. This analysis was run in the SAS mixed procedure (SAS Institute, Inc.) and the B-splines were calculated using the $n s($ ) command in Splus v8.0 (Insightful Corp). A random intercept was included in all models and a random slope was modeled using natural cubic B-splines. Akaike's Information Criterion was used to determine the final model [15]. We allowed a maximum of 5 and 3 degrees of freedom (df) for the fixed and random effect B-splines, respectively, and the final model included the maximum, resulting in the most flexible model. The same flexible analysis was used to model CD4+ T-cell counts over time. The final model included 5 and $1 \mathrm{df}$ for the fixed and random effects, respectively.

As VL was measured using different assays, the lower limit of detection varied. Six limits exist in our data: $<20$, $20-50,<50,<200,200-400$, and $<400$. In preparation for the B-spline analyses, we imputed plasma HIV RNA copies/mL such that those with (a) $<20$ were set to 10 , (b) 20-50 to 35 , (c) $<50$ to the mean of all values $<50$ (real and imputed), (d) $<200$ to the mean of all values below 200 (real and imputed), (e) $200-400$ to 300 , and (f) $<400$ to the mean of all values $<400$ (real and imputed). The $\log _{10}$ transformation was conducted after the imputation.

\section{Results}

3.1. Demographics and HIV Disease Characteristics of the Study Population. Medical records were available for 117 of 123 pregnancies that met inclusion criteria. These involved 12 women with 2 pregnancies (Table 1). The median age at delivery was 30 years. Thirty-one patients (30\%) were black and $41(39 \%)$ were Hispanic. A third of patients most likely acquired HIV in a foreign country.

The most common risk factor for HIV acquisition was heterosexual intercourse, reported by 97 patients (92\%). In 70 patients $(67 \%)$, the diagnosis of HIV infection was established before pregnancy. Of the 35 patients diagnosed with HIV during pregnancy, 11 (34\%) were diagnosed during the first trimester, $16(50 \%)$ during the second trimester, and $5(16 \%)$ during the third trimester. 
TABle 1: Demographics and baseline characteristics of HIV infection.

\begin{tabular}{|c|c|}
\hline Characteristic & $N$ or Median ( $\%$ or IQR) \\
\hline Patients & 105 \\
\hline One pregnancy with CHIP & $93(89)$ \\
\hline Two pregnancies with CHIP & $12(11)$ \\
\hline Maternal age at delivery & $30(26,34)$ \\
\hline \multicolumn{2}{|l|}{ Race } \\
\hline White & $72(68.6)$ \\
\hline Black & $31(29.5)$ \\
\hline Other & $2(1.9)$ \\
\hline \multicolumn{2}{|l|}{ Ethnicity } \\
\hline Hispanic & $41(39)$ \\
\hline Not Hispanic & $64(61)$ \\
\hline \multicolumn{2}{|l|}{ Country of origin } \\
\hline African country* & $15(14)$ \\
\hline Mexico & $17(16)$ \\
\hline USA & $66(63)$ \\
\hline Other & $4(4)$ \\
\hline Unknown & $3(3)$ \\
\hline \multicolumn{2}{|l|}{ HIV risk factors ${ }^{\dagger}$} \\
\hline IV drug use & $12(11)$ \\
\hline Heterosexual sex & $97(92)$ \\
\hline Transfusion & $5(5)$ \\
\hline \multicolumn{2}{|l|}{ HIV diagnosis } \\
\hline Prior to first pregnancy at CHIP & $70(67)$ \\
\hline During pregnancy & $35(33)$ \\
\hline \multicolumn{2}{|l|}{ ARV experience } \\
\hline Naive & $51(44)$ \\
\hline $\begin{array}{l}\text { Experienced on therapy at the } \\
\text { onset of pregnancy }\end{array}$ & $29(25)$ \\
\hline $\begin{array}{l}\text { Experienced off therapy at the } \\
\text { onset of pregnancy }\end{array}$ & $30(26)$ \\
\hline Unknown & $7(5)$ \\
\hline Plasma HIV RNA at first visit & 109 pregnancies \\
\hline Median (range) & $2657(225,16700)$ \\
\hline$>400$ copies $/ \mathrm{mL}$ & $78(72)$ \\
\hline CD4+ count at first visit & 108 pregnancies \\
\hline Median (range) & $450(269,628)$ \\
\hline$<200$ cells $/ \mu \mathrm{L}$ & $13(12 \%)$ \\
\hline
\end{tabular}

* Includes Ethiopia, Liberia, Nigeria, Kenya, and Somalia.

${ }^{\dagger}$ Nine patients have more than one risk factor.

At the first pregnancy visit, the median VL was 2657 copies $/ \mathrm{mL}$ including $78(72 \%)$ with $>400$ copies $/ \mathrm{mL}$. The median CD4+ T-cells was 450 cells/ $\mu \mathrm{L} ; 13$ women $(12 \%)$ had $<200$ cells $/ \mu \mathrm{L}$. In 51 pregnancies $(44 \%)$, the women were ARV naïve and in 59 (50\%) ARV experienced, including 29 patients who became pregnant on therapy.

3.2. Pregnancy Outcome. Median gestational age at delivery was 38 weeks (interquartile range $(I Q R)=37-40$ weeks).
TABLE 2: Adherence to the last ARV regimen used during pregnancy.

\begin{tabular}{lc}
\hline ARV regimen & $N$ with $\geq 95 \%$ adherence/ $N$ total $(\%)$ \\
\hline Any regimen & $36 / 44(82)$ \\
$\geq 3$-drug with PI & $25 / 28(89)$ \\
$\geq 3$-drug with NNRTI & $3 / 5(60)$ \\
$\geq 3$-drug with PI and & $3 / 5(60)$ \\
NNRTI & $5 / 6(83)$ \\
NRTI only & \\
\hline
\end{tabular}

Thirty-six women (31\%) delivered by scheduled Cesarean section. The 114 single and 3 twin pregnancies resulted in, 117 infants without HIV infection, 1 fetal demise, 1 stillbirth and 1 neonate who died of sepsis at 1 day of life. Of the 117 neonates who lived $>1$ day, $98(84 \%)$ received AZT and 19 (16\%) HAART. These infants were not infected with HIV. The incidence of MTCT was 0 with a $95 \%$ confidence interval $=0 \%$ to $3 \%$.

3.3. ARV Utilization during Pregnancy. At delivery, 106/114 (94\%) pregnant women were on HAART ( $\geq 3$ drugs from $\geq 2$ classes); 7 women (4\%), who did not tolerate PIs or NNRTIs, were on 2 or 3 NRTIs; and 1 woman, who delivered shortly after referral precluding a change in therapy, was on AZT monotherapy. The most common drugs at delivery were AZT $(N=89)$, lamivudine $(N=108)$, stavudine $(N=22)$, nevirapine (NVP; $N=19$ ), nelfinavir (NFV; $N=55)$, and $\operatorname{LPVr}(N=25)$.

The median duration of continuous therapy up to delivery was 22 weeks (IQR of 15 to 35 weeks). Among 84 women who started ARV during pregnancy after being off therapy for several months or never being on therapy, the median duration of therapy during pregnancy was 20 weeks (IQR of 11 to 25 weeks). Of these, 77 (92\%) received ARV for $\geq 4$ weeks and $61(73 \%)$ for $\geq 12$ weeks.

3.4. Adherence. Adherence was measured by pill counts, pharmacy refill histories, MEMS caps, and detailed interviews in 44 pregnancies. Of these, 36 women (82\%) took $\geq 95 \%$ doses of the prescribed medication, 7 (16\%) took $50 \%$ doses-95\%, and 1 took $<50 \%$ doses. The analysis of adherence by ARV regimen (Table 2) did not reveal any differences across regimens.

3.5. Psychosocial Support. This was provided in 76 of 117 pregnancies. A median of 7 support interventions per pregnancy took place $(\mathrm{IQR}=4$ to 13$)$. These included case management visits $(N=59)$, referrals to housing agencies $(N=31)$ and peer advocacy groups $(N=30)$, food $(N=59)$ and financial $(N=56)$ assistance, and psychoeducational group activities $(N=37)$.

3.6. Virologic Response to ARV during Pregnancy. For all study participants, the median VL at delivery was 51 copies $/ \mathrm{mL}$ (IQR $<20$ to $292 ; N=95$ ), which represented a significant decrease compared with the first pregnancy visit median of 2657 copies/mL $(P<.0001)$. At the last visit before delivery, 


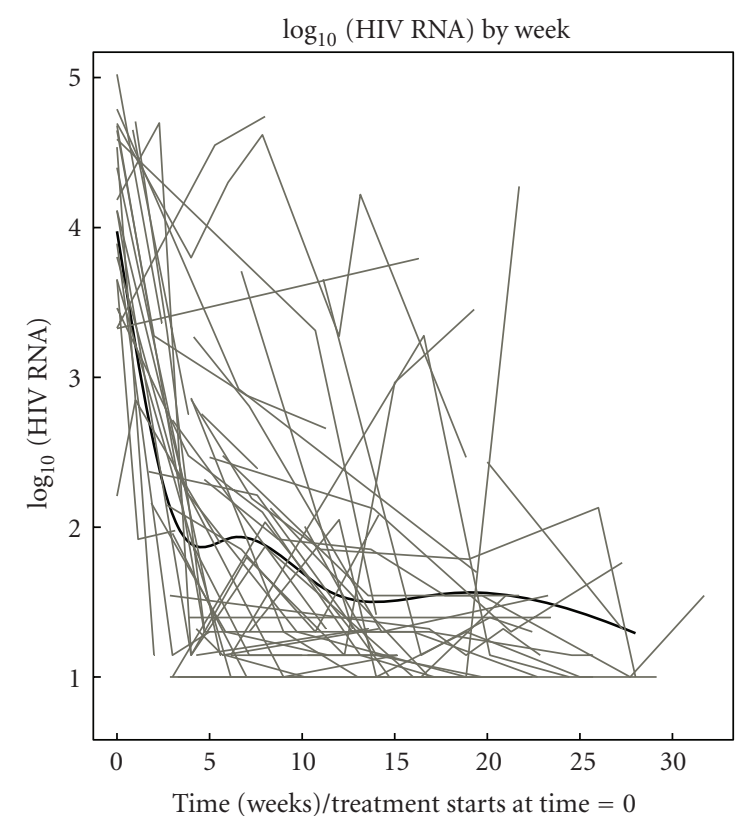

FIgURE 1: Kinetics of the plasma HIV RNA in pregnant women on HAART. Data were derived from 76 pregnant women who initiated or reinitiated HAART during pregnancy. 0 weeks indicates the beginning of therapy. Grey lines represent individual plasma HIV RNA trajectories. The black line represents the population curve generated by the mixed-model analysis. The predictions of the model were confirmed by additional analyses which showed that at 4 weeks of HAART, $42 \%$ of mothers had $<100$ HIV RNA copies/mL of plasma; at 12 weeks, $42 \%$ had $<50$ copies $/ \mathrm{mL}$; and at 24 weeks, $56 \%$ had $<50$ copies $/ \mathrm{mL}$. However, it should be noted that the model's estimated rise between 5 and 7 weeks is likely not real.

80 women $(82 \%)$ had plasma HIV RNA $<400$ copies/mL and 53 women $(56 \%)$ had $<50$ HIV RNA copies/mL.

To determine the kinetics of the virologic response to HAART during pregnancy, we applied a mixed-model analysis to data from 76 of 84 patients who initiated or reinitiated HAART during pregnancy and had multiple tests during pregnancy (Figure 1). The model, which uses all available data to estimate the mean VL trajectory over time, estimated that after 4 weeks of HAART the $\log _{10}$ VL decreased by $\sim 2 \operatorname{logs}$ from baseline to a back-transformed mean $<100$ copies/mL. Both at 12 and 24 weeks of HAART, the estimated back-transformed mean VL was $<50$ copies/mL. We verified the predictions made by the model by calculating the proportions of subjects with VL $<100$ copies/mL at 4 weeks and $<50$ copies/mL at 12 and 24 weeks of HAART. Of 39 women with VL measurements at 4 weeks of HAART, $15(42 \%)$ had $<100$ copies/mL; at 12 weeks of HAART, 11 of 26 women $(42 \%)$ had $<50$ copies/mL; and at 24 weeks of HAART, 9 of 16 women $(56 \%)$ had $<50$ copies $/ \mathrm{mL}$. These data confirmed the predictions of the model.

3.7. Immunologic Response to $A R V$ during Pregnancy. At delivery, the median CD4+ T-cell number was 540 cells $/ \mu \mathrm{L}$ (IQR of 416 to $678 ; N=92$ ): $92 \%$ of values $>200$ cells $/ \mu \mathrm{L}$. The median CD4+ T-cell increase between first pregnancy visit and delivery was 88 cells $/ \mu \mathrm{L}(P<.0001$ by signed-rank test; $N=92$ ).

In the mixed-model analysis used to elucidate the kinetics of CD4+ T-cells during pregnancy, we set the reference time at delivery to take into account the upward effect of ARV and the downward effect of pregnancy on the CD4+ T-cells. The model indicated that the CD4+ T-cells started to increase at 6 weeks prior to delivery and peaked at approximately 8 weeks after delivery in all subjects (Figure 2(a)) and in those who initiated or reinitiated HAART during pregnancy (Figure 2(b)). Among 54 women with postpartum data, there were no appreciable differences in postpartum CD4+ T-cell numbers of 36 women who continued therapy for $\geq 12$ weeks, compared with those of 18 women who discontinued therapy immediately after delivery. An additional mixedmodel analysis allowed for separate CD4+ T-cell trajectories postdelivery for the 18 women who discontinued therapy immediately after delivery and the 36 women who continued therapy for $\geq 12$ weeks. From this model, the estimated CD4+ T-cell count at 12 weeks postpartum was 763 and 679, for women who stopped therapy and continued therapy for $\geq 12$ weeks, respectively, a difference of $84(P=.29)$.

3.8. PI Plasma Concentrations during Pregnancy. Thirtytwo pregnant women had $\geq 1$ PI TDM for a total of 92 measurements. TDM was performed at a median of 5.5 weeks after initiation or change in therapy (IQR of 3.5 to 30 weeks). Eighty-two TDM assays were performed in women taking PI doses recommended for nonpregnant adults (regular) and 10 in women taking increased doses.

Table 3 depicts the TDM results from women on regular PI doses. There were 7 measurements in the 1st trimester. Of these, $4(57 \%)$ were within the targeted range (normal), 2 (29\%) were low, and 1 (14\%) was high. The 57 TDM assays from the second trimester included 34 (60\%) normal, 20 (35\%) low, and 3 (5\%) high results. The 18 TDM assays from the 3rd trimester included 9 (50\%) normal, 8 (44\%) low, and $1(6 \%)$ high results. Across all gestational ages, $57 \%$ of the TDM results were normal, 37\% low and $6 \%$ high. The distribution of TDM results did not significantly differ across trimesters (Fisher's exact test, $P=.70$ ).

The analysis of the plasma concentrations achieved by the most commonly used PI in this study, LPVr, NFV, and saquinavir (SAQr), showed that $75 \%$ of all LPV measurements were normal and 25\% were low across all trimesters; $20 \%$ to $59 \%$ of the NFV levels were normal with a trend of decreasing concentrations from 1 st to 3rd trimester; and 30 to $50 \%$ of SAQ concentrations were normal.

There were 10 PI TDM results during the 2 nd or 3 rd trimester in women who received increased PI doses (6 received LPVr 533/133 mg bid and 3 NFV $1500 \mathrm{mg}$ bid). Of these, 3 measurements (30\%) were below target.

3.9. Factors That Affected Virologic Response to Therapy during Pregnancy. To evaluate potential differences between HAART regimens with respect to VL decay during pregnancy, the mixed-model analysis of $\log _{10}$ VL described above (Virologic response to therapy) was used to compare NFV- 


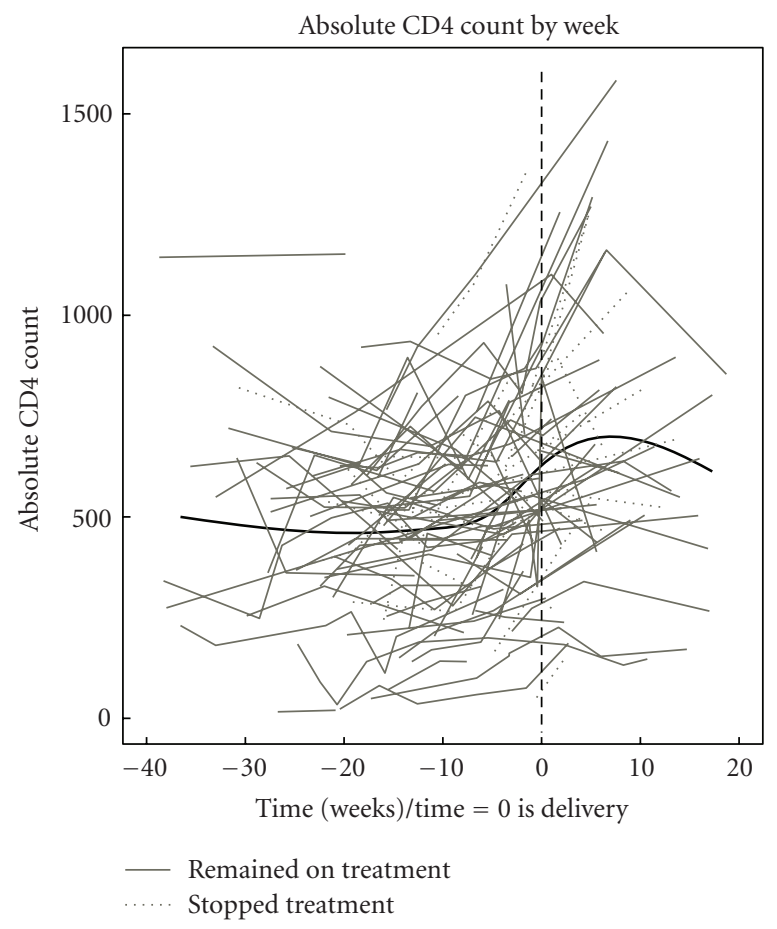

(a)

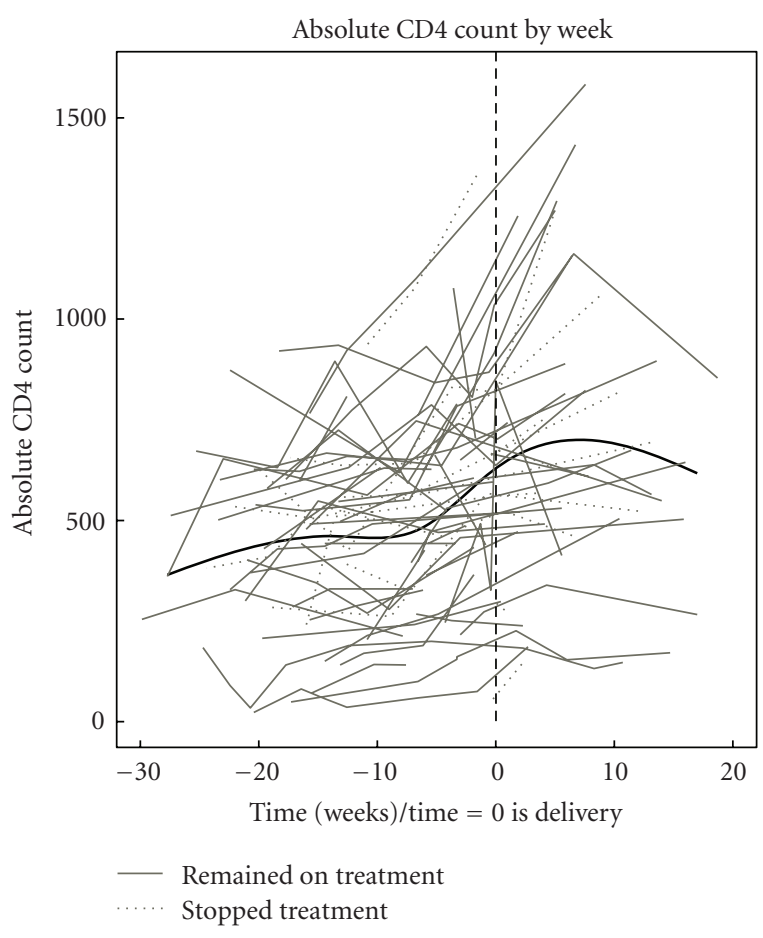

(b)

FIGURE 2: Kinetics of CD4+ counts in HIV-infected pregnant women on combination ARV. (a) Data were derived from 100 pregnant with available data who started therapy before or during pregnancy. (b) Data were derived from 76 pregnant women who started therapy during pregnancy after being off therapy for several months or never being on therapy. Time 0 indicates delivery. Grey lines represent individual CD4+ trajectories. The black line represents the population curve generated by the mixed-model analysis.

TABLE 3: Distribution of therapeutic drug monitoring results of protease inhibitors during pregnancy.

\begin{tabular}{ccccccc}
\hline & All & LPVr & NFV & SAQr & IDVr & RTV \\
\hline 1st trimester & 7 & 4 & 2 & 1 & 0 & 0 \\
Low & $2(29)$ & $1(25)$ & 1 & 0 & 0 & 0 \\
Normal & $4(57)$ & $3(75)$ & 1 & 0 & 0 & 0 \\
High & $1(14)$ & 0 & 0 & 1 & 0 & 0 \\
2nd trimester & 57 & 28 & 17 & 10 & 0 & 2 \\
Low & $20(35)$ & $7(25)$ & $6(35)$ & $5(50)$ & 0 & 2 \\
Normal & $34(60)$ & $21(75)$ & $10(59)$ & $3(30)$ & 0 & 0 \\
High & $3(5)$ & 0 & $1(6)$ & $2(20)$ & 0 & 0 \\
3rd trimester & 18 & 5 & 5 & 4 & 2 & 2 \\
Low & $8(44)$ & $1(20)$ & $4(80)$ & $2(50)$ & 1 & 0 \\
Normal & $9(50)$ & $4(75)$ & $1(20)$ & $2(50)$ & 0 & 2 \\
High & $1(6)$ & 0 & 0 & 0 & 1 & 0 \\
\hline
\end{tabular}

Numbers (\%) represent pregnant women taking PI doses recommended for adults.

Abbreviations: IDVr = indinavir/ritonavir; LPVr = lopinavir/ritonavir; NFV = nelfinavir; RTV = ritonavir; SAQr = saquinavir/ritonavir.

$(N=49)$ versus LPVr-containing HAART $(N=20)$. Differences in estimated $\log _{10}$ VL between the regimens, calculated as NFV estimate minus LPVr estimate, were 0.01
$(P=.96), 0.44(P=.10), 0.39(P=.12)$, and $0.09(P=.78)$ at $4,8,12$, and 24 weeks on treatment, respectively.

An analysis of the factors that could potentially affect the response to therapy defined by undetectable VL at delivery, including race, ethnicity, country of origin (as a surrogate of HIV-1 clade), magnitude of VL before initiation of therapy, ARV experience, HIV drug resistance (data presented elsewhere) [16], adherence, TDM, and utilization of psychosocial support services, was performed (Table 4). The data revealed significant associations only for adherence $(P=.006)$ and psychosocial support $(P=.010)$.

\section{Discussion}

To determine what length of HAART administration during pregnancy was needed to achieve undetectable VL, we analyzed the kinetics of the virologic response in women who started HAART during pregnancy. The time to undetectable VL did not differ from that previously reported in HIVinfected nonpregnant adults $[17,18]$, with a majority of pregnant women achieving VL $<50$ copies/mL after 12 to 24 weeks of therapy. The corollary of this observation is that pregnant women who start HAART during or before the 2nd trimester are likely to achieve VL $<50$ copies $/ \mathrm{mL}$ at delivery. In contrast, women starting HAART less than 4 weeks before the anticipated date of delivery are likely candidates to scheduled Cesarean sections. 
TABLE 4: Relationship between virologic response to ARV during pregnancy and selective factors that may affect this response.

\begin{tabular}{lccc}
\hline Factor & $\begin{array}{c}N(\%) \\
\text { undetectable }\end{array}$ & $\begin{array}{c}N(\%) \\
\text { detectable }\end{array}$ & $P$ value \\
\hline Country of origin & $39(80)$ & $10(20)$ & .16 \\
$\quad$ US & $11(79)$ & $3(21)$ & \\
$\quad$ Mexico & $15(100)$ & 0 & \\
$\quad$ African country & & & \\
HIV plasma RNA at & & & \\
first visit & $6(75)$ & $2(25)$ & .63 \\
$\quad \geq 50,000$ copies/mL & $72(83)$ & $15(17)$ & \\
$\quad<50,000$ copies/mL & & & .27 \\
Prepregnancy ARV & $33(77)$ & $10(23)$ & \\
$\quad$ Naive & $40(87)$ & $6(13)$ & \\
$\quad$ Experienced & & & \\
TDM & $53(79)$ & $14(21)$ & \\
Performed & $25(89)$ & $3(11)$ & \\
$\quad$ Not performed & & & .006 \\
Adherence & $1(100)$ & 0 & \\
$<50 \%$ & $3(50)$ & $3(50)$ & \\
50\% to 95\% & $29(94)$ & $2(6)$ & \\
$\quad>95 \%$ & & & \\
Psychosocial support & $63(89)$ & $8(11)$ & \\
Utilized & $15(63)$ & $9(37)$ & \\
Not utilized & & & \\
\hline
\end{tabular}

In our patient population, the rate of Cesarean section was similar to that reported for other areas in the US and lower than that reported in Europe, where the use of scheduled Cesarean section is twice as common as in the US $[1,19]$. However, MTCT in this study was lower than that documented in contemporaneous studies in the US $[4,19]$ and similar to that reported in Europe $[1,3]$. These differences may be explained by the fact that $94 \%$ of HIVinfected pregnant women in this study received HAART for PMTCT, which is a higher proportion than the rate of $50 \%$ to $70 \%$ documented in other studies [2-5]. Taken together, these observations suggest that high uptake of HAART may decrease the need of scheduled Cesarean sections for PMTCT $[2,3]$ without compromising protection of the neonate against HIV infection.

The analysis of the kinetics of CD4+ T-cells in pregnancy revealed a delay in the response to HAART compared with the kinetics of VL. In pregnancy, the most significant increases in CD4+ T-cell numbers occurred in the last 6 weeks of pregnancy and first 6 weeks of postpartum. This pattern was observed in women who started HAART before or during pregnancy and was independent of continuing HAART post-partum. This pattern is most likely due to physiologic changes of pregnancy, including the increased volume of distribution of white blood cells and/or hormonal changes depressing CD4+ T-cells. The implication of this observation is that a lack of increase of CD4+ T-cells in pregnant women may not indicate virologic failure.
An important question in the care of HIV-infected pregnant women is whether there is an ARV regimen more likely than others to rapidly decrease the VL in women diagnosed with HIV late in pregnancy. We did not find any significant differences in the kfinetics of VL as a function of the PI used in HAART. Previous studies showed that nonpregnant HIV-infected adults on NFV-containing regimens were less likely to achieve or maintain viral suppression compared with individuals receiving LPVr-containing regimens [20]. However, during the limited duration of therapy for PMTCT, this difference may not be relevant. These data suggest that although HAART is critical in order to achieve a satisfactory virologic response during pregnancy, the choice of PI has secondary importance with respect to virologic response. Patel et al. showed that NVP-based regimens are associated with faster decay of VL compared with NFV-based regimens [10]. In this study, the number of women who intitiated NVP during pregnancy was too low for a formal analysis. Further studies using NNRTIs that do not carry the same high risk of serious adverse events as NVP are warranted to determine whether an NNRTI-based regimen may be advantageous when therapy is initiated late in pregnancy.

With respect to plasma concentrations achieved by various PIs during pregnancy, we found that LPVr levels were below target in $20 \%$ to $25 \%$ of pregnant women on adult recommended doses across all trimesters, whereas for another PI, $35 \%$ to $80 \%$ of the levels were below target. Others have shown that up to $72 \%$ of women on $\mathrm{LPVr}$ do not reach target concentrations of LPV during the 3rd trimester [11, 12, 21]. Acosta et al. found SAQ plasma levels uniformly above target [22] during pregnancy, whereas in our study $50 \%$ of subjects had levels below target. Our findings on NFV plasma concentrations are in accordance with those of Nellen et al. who also found that $51 \%$ of pregnant women did not reach target concentrations [23]. Differences in drug concentrations across studies may be accounted by technical differences, effects of race and ethnicity on drug metabolism, and compliance with therapy. A novel contribution of our study is to reveal that plasma PI concentrations are often below target not only in the last trimester of pregnancy but also earlier in pregnancy. This suggests that other factors, in addition to the increased volume of distribution of the drugs, may contribute to this effect. The practical implication of our findings is to underscore the importance of performing TDM or other pharmacokinetic studies during the 1st and 2nd trimesters of pregnancy and also after each dose modification, since 30\% of the pregnant women on increased PI doses had plasma drug levels below target.

The use of TDM did not significantly affect the virologic response to therapy in this study. Some studies in nonpregnant HIV-infected adults showed improved virologic response to PI-containing regimens in subjects whose doses were adjusted based on TDM results [24-26], but one study failed to show any benefit [27]. This last study had a period of followup of only 12 weeks. Likewise, it may be difficult to assess the benefit of TDM in pregnancy due to the limited duration of therapy. Nevertheless, it is reasonable to assume that it would be undesirable to administer drugs at 
subtherapeutic levels to control a virus that has the ability to quickly develop drug resistance mutations [28], particularly if ARV continues to be administered after pregnancy. Further studies are warranted to determine the effect of TDM on the development of resistance to ARV.

Among multiple factors that could potentially affect virologic response to therapy, suppression of viral replication at delivery was positively associated with adherence, confirming previous observations [29]. We did not find significant differences in adherence between patients receiving once daily versus twice daily regimens or PI-containing versus sparing regimens, which is a novel finding, perhaps specific of pregnancy and has important implications for the clinical practice. Overall, $\geq 95 \%$ adherence was recorded in $82 \%$ of the women in this study, which is consistent with other reports [30].

Utilization of psychosocial support services was significantly associated with virologic response to therapy during pregnancy, which makes intuitive sense, but has not been previously demonstrated in a formal analysis. Although the association between utilization of psychosocial services and virologic success may indicate that women who are committed to medical treatment for PMTCT are also likely to seek resources to improve other aspects of their lives, it may also indicate that support services facilitate adherence to therapy [30-32].

In summary, undetectable VL at delivery was associated with lack of vertical transmission of HIV in this group of women who predominantly delivered by vaginal route. Undetectable VL was achieved as soon as 4 weeks after initiation of HAART in $50 \%$ of the patients and at 12 weeks in the vast majority. Achieving undetectable VL at delivery did not depend on the specific PIs or NNRTIs included in the treatment regimen. Adherence, which was important to reach undetectable VL at delivery, did not vary with the drugs included in the treatment regimen, either. The pattern that emerges is that critical factors for PMTCT without high utilization of Cesarean sections are initiating therapy $\geq 12$ weeks prior to delivery with a regimen that is well tolerated and conducive to $\geq 95 \%$ adherence.

\section{Acknowledgments}

The authors thank the following providers for referring their patients to our service: L. Anneberg, M.D.; B. Barber, N.P.; C. Benson, M.D.; W. Burman, M.D.; W. Callan, M.D.; P. Caraway, N.P.; M. Carten, M.D.; A. Davis, M.D.; J. DesJardin, M.D.; A. Dulit, M.D.; C. Fisher, M.D.; R. Gass, M.D.; D. Guinn, M.D.; W. Hoppe, M.D.; S. Johnson, M.D.; D. Kronbach, M.D.; C. Kurwola, M.D.; N. Madinger, M.D.; S. Mason, M.D.; L. McLaughlan, M.D.; R. Peskind, M.D.; H. Puget, M.D.; K. Rowley, M.D.; M. Schulte, M.D.; M. Schwarz, M.D.; J. Sheppard, M.D.; J. Shlay, M.D.; B. St. Dennis, R.N.; D. Stark, M.D.; C. Steinberg, M.D.; C. Stevens, M.D.; D. Strandberg, M.D.; D. Urioste, M.D.; W. Williams, M.D.; M. Winslow, M.D.; T. Yettern, N.P.; and B. Young, M.D. This research was supported by research grants from Abbott
Laboratories, Inc. and from Pfizer, Inc. CHIP has funding from Ryan White title IV (2H12HA0070-07 to MJL) and NICHD (U0132915-05 to MJL).

\section{References}

[1] C. Thorne, "Mother-to-child transmission of HIV infection in the era of highly active antiretroviral therapy," Clinical Infectious Diseases, vol. 40, no. 3, pp. 458-465, 2005.

[2] C. L. Townsend, M. Cortina-Borja, C. S. Peckham, A. de Ruiter, H. Lyall, and P. A. Tookey, "Low rates of motherto-child transmission of HIV following effective pregnancy interventions in the United Kingdom and Ireland, 20002006," AIDS, vol. 22, no. 8, pp. 973-981, 2008.

[3] J. Warszawski, R. Tubiana, J. Le Chenadec, et al., "Mother-tochild HIV transmission despite antiretroviral therapy in the ANRS French Perinatal Cohort," AIDS, vol. 22, no. 2, pp. 289299, 2008.

[4] E. R. Cooper, M. Charurat, D. N. Burns, W. Blattner, and R. Hoff, "Trends in antiretroviral therapy and mother-infant transmission of HIV. The Women and Infants Transmission Study Group," Journal of Acquired Immune Deficiency Syndromes, vol. 24, no. 1, pp. 45-47, 2000.

[5] A. Dorenbaum, C. K. Cunningham, R. D. Gelber, et al., "Two-dose intrapartum/newborn nevirapine and standard antiretroviral therapy to reduce perinatal HIV transmission: a randomized trial," Journal of the American Medical Association, vol. 288, no. 2, pp. 189-198, 2002.

[6] J. P. A. Ioannidis, E. J. Abrams, A. Ammann, et al., "Perinatal transmission of human immunodeficiency virus type 1 by pregnant women with RNA virus loads < 1000 copies/mL," Journal of Infectious Diseases, vol. 183, no. 4, pp. 539-545, 2001.

[7] C. N. Hudson, "Elective caesarean section for prevention of vertical transmission of HIV-1 infection," The Lancet, vol. 353, no. 9158, pp. 1030-1031, 1999.

[8] G. Jourdain, J.-Y. Mary, S. Le Coeur, et al., "Risk factors for in utero or intrapartum mother-to-child transmission of human immunodeficiency virus type 1 in Thailand," Journal of Infectious Diseases, vol. 196, no. 11, pp. 1629-1636, 2007.

[9] D. K. Ekouevi, A. Inwoley, B. Tonwe-Gold, et al., "Variation of CD4 count and percentage during pregnancy and after delivery: implications for HAART initiation in resourcelimited settings," AIDS Research and Human Retroviruses, vol. 23, no. 12, pp. 1469-1474, 2007.

[10] D. Patel, M. Cortina-Borja, C. Thorne, et al., "Time to undetectable viral load after highly active antiretroviral therapy initiation among HIV-infected pregnant women," Clinical Infectious Diseases, vol. 44, no. 12, pp. 1647-1656, 2007.

[11] K. Manavi, A. McDonald, and A. Al-Sharqui, "Plasma lopinavir trough levels in a group of pregnant women on lopinavir, ritonavir, zidovudine, and lamivudine," AIDS, vol. 21, no. 5, pp. 643-645, 2007.

[12] A. M. Stek, M. Mirochnick, E. Capparelli, et al., "Reduced lopinavir exposure during pregnancy," AIDS, vol. 20, no. 15, pp. 1931-1939, 2006.

[13] J. Ghosn, I. De Montgolfier, C. Cornélie, et al., “Antiretroviral therapy with a twice-daily regimen containing 400 milligrams of indinavir and 100 milligrams of ritonavir in human immunodeficiency virus type 1-infected women during pregnancy," Antimicrobial Agents and Chemotherapy, vol. 52, no. 4, pp. 1542-1544, 2008. 
[14] H. Liang, H. Wu, and R. J. Carroll, "The relationship between virologic and immunologic responses in AIDS clinical research using mixed-effects varying-coefficient models with measurement error," Biostatistics, vol. 4, no. 2, pp. 297 312, 2003.

[15] R. C. Littell, G. A. Milliken, W. W. Stroup, and R. D. Wolfinger, SAS System for Mixed Models, SAS Institute, 1996.

[16] A. Weinberg, J. Forster-Harwood, E. J. McFarland, et al., "Resistance to antiretrovirals in HIV-infected pregnant women," Journal of Clinical Virology, vol. 45, no. 1, pp. 39-42, 2009.

[17] M. Gartland, "AVANTI 3: a randomized, double-blind trial to compare the efficacy and safety of lamivudine plus zidovudine versus lamivudine plus zidovudine plus nelfinavir in HIV-1infected antiretroviral-naive patients," Antiviral Therapy, vol. 6, no. 2, pp. 127-134, 2001.

[18] R. L. Murphy, S. Brun, C. Hicks, et al., "ABT-378/ritonavir plus stavudine and lamivudine for the treatment of antiretroviralnaive adults with HIV-1 infection: 48-week results," AIDS, vol. 15, no. 1, pp. F1-F9, 2001.

[19] M. Nan, N. Harris, M. Lisa, et al., "Enhanced perinatal surveillance- United States, 1999-2001," Special Surveillance Report 4, US Department of Health and Human Services, Centers for Disease Control and Prevention, Atlanta, Ga, USA, 2004.

[20] S. Walmsley, B. Bernstein, M. King, et al., "Lopinavir-ritonavir versus nelfinavir for the initial treatment of HIV infection," The New England Journal of Medicine, vol. 346, no. 26, pp. 2039-2046, 2002.

[21] A. Gingelmaier, M. Kurowski, R. Kästner, et al., "Placental transfer and pharmacokinetics of lopinavir and other protease inhibitors in combination with nevirapine at delivery," AIDS, vol. 20, no. 13, pp. 1737-1743, 2006.

[22] E. P. Acosta, A. Bardeguez, C. D. Zorrilla, et al., "Pharmacokinetics of saquinavir plus low-dose ritonavir in human immunodeficiency virus-infected pregnant women," Antimicrobial Agents and Chemotherapy, vol. 48, no. 2, pp. 430-436, 2004.

[23] J. F. J. B. Nellen, I. Schillevoort, F. W. N. M. Wit, et al., "Nelfinavir plasma concentrations are low during pregnancy," Clinical Infectious Diseases, vol. 39, no. 5, pp. 736-740, 2004.

[24] D. Burger, P. Hugen, P. Reiss, et al., "Therapeutic drug monitoring of nelfinavir and indinavir in treatment-naive HIV-1-infected individuals," AIDS, vol. 17, no. 8, pp. 11571165, 2003.

[25] J. Durant, P. Clevenbergh, R. Garraffo, et al., "Importance of protease inhibitor plasma levels in HIV-infected patients treated with genotypic-guided therapy: pharmacological data from the Viradapt Study," AIDS, vol. 14, no. 10, pp. 1333$1339,2000$.

[26] J. Durant, P. Clevenbergh, P. Halfon, et al., "Drug-resistance genotyping in HIV-1 therapy: the VIRADAPT randomised controlled trial," The Lancet, vol. 353, no. 9171, pp. 2195-2199, 1999.

[27] P. Clevenbergh, R. Garraffo, J. Durant, and P. Dellamonica, "PharmAdapt: a randomized prospective study to evaluate the benefit of therapeutic monitoring of protease inhibitors: 12 week results," AIDS, vol. 16, no. 17, pp. 2311-2315, 2002.

[28] C. J. L. La Porte, D. J. Back, T. Blaschke, et al., "Updated guideline to perform therapeutic drug monitoring for antiretroviral agents," Reviews in Antiviral Therapy, vol. 2006, no. 3, pp. 414, 2006.

[29] J. McNabb, J. W. Ross, K. Abriola, C. Turley, C. H. Nightingale, and D. P. Nicolau, "Adherence to highly active antiretroviral therapy predicts virologic outcome at an inner-city human immunodeficiency virus clinic," Clinical Infectious Diseases, vol. 33, no. 5, pp. 700-705, 2001.

[30] A. D. Bardeguez, J. C. Lindsey, M. Shannon, et al., "Adherence to antiretrovirals among US women during and after pregnancy," Journal of Acquired Immune Deficiency Syndromes, vol. 48, no. 4, pp. 408-417, 2008.

[31] S. C. J. M. Vervoort, J. C. C. Borleffs, A. I. M. Hoepelman, and M. H. F. Grypdonck, "Adherence in antiretroviral therapy: a review of qualitative studies," AIDS, vol. 21, no. 3, pp. 271$281,2007$.

[32] L. Osterberg and T. Blaschke, "Adherence to medication," The New England Journal of Medicine, vol. 353, no. 5, pp. 487-497, 2005. 


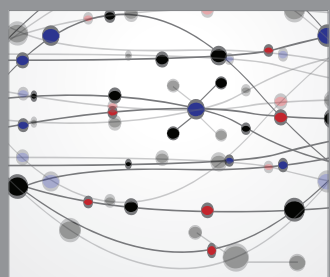

The Scientific World Journal
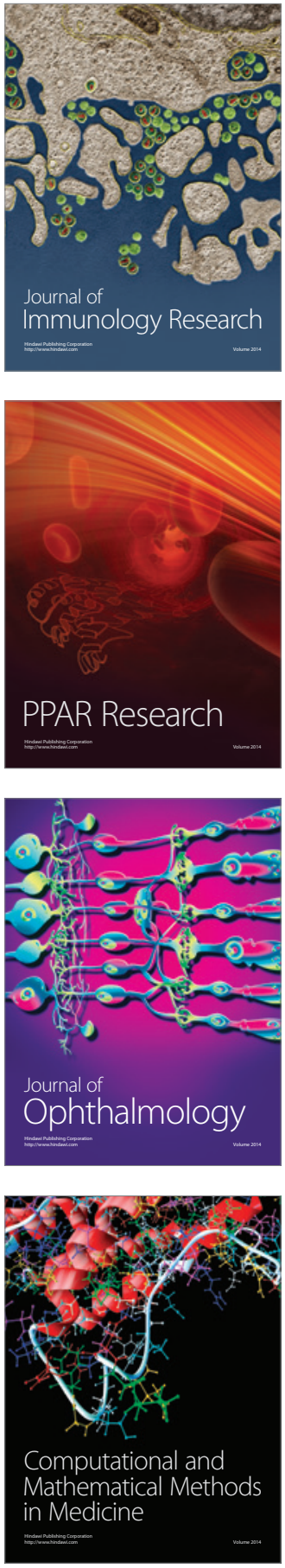

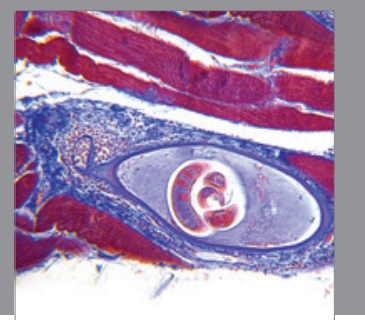

Gastroenterology

Research and Practice
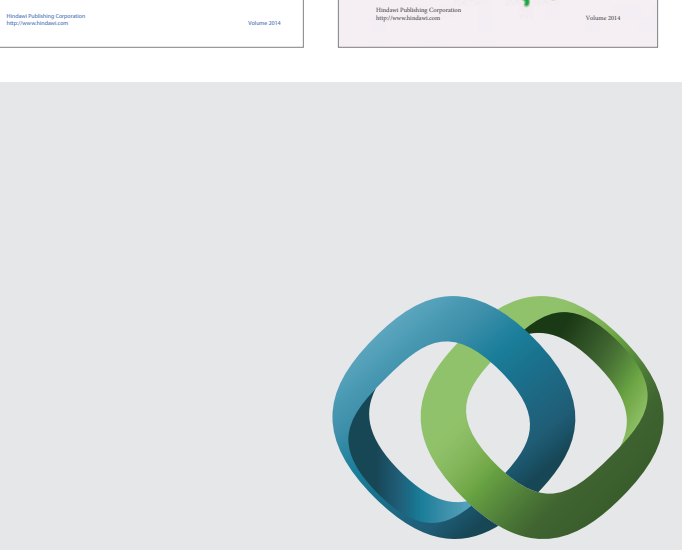

\section{Hindawi}

Submit your manuscripts at

http://www.hindawi.com
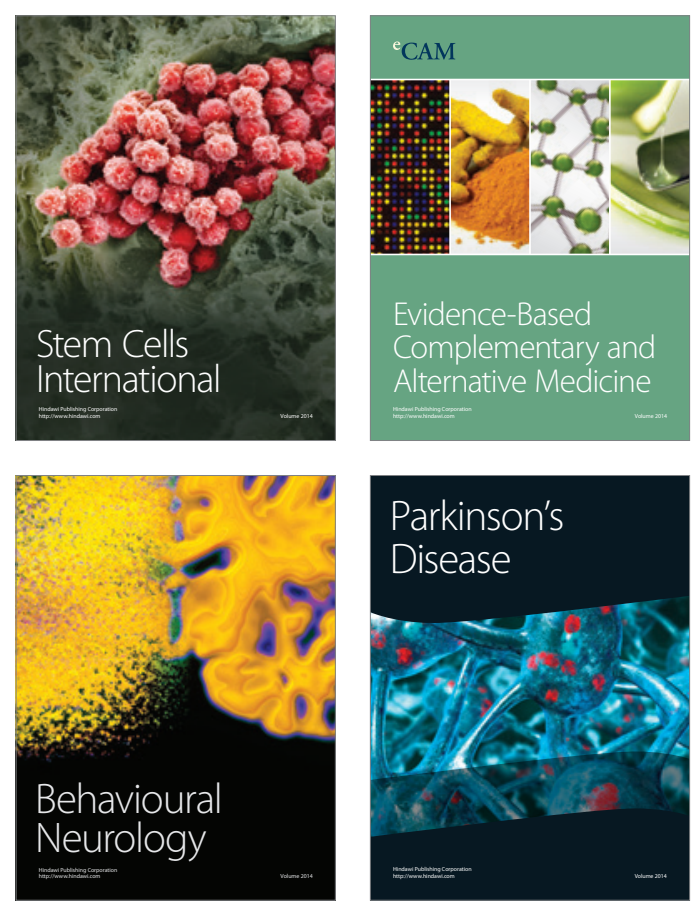

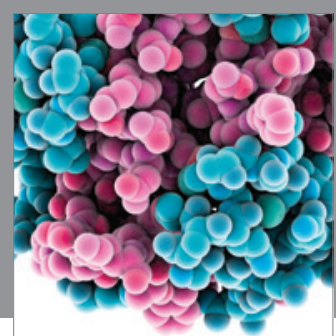

Journal of
Diabetes Research

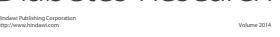

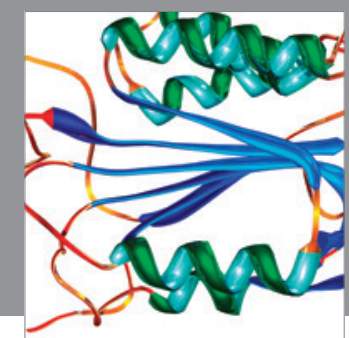

Disease Markers
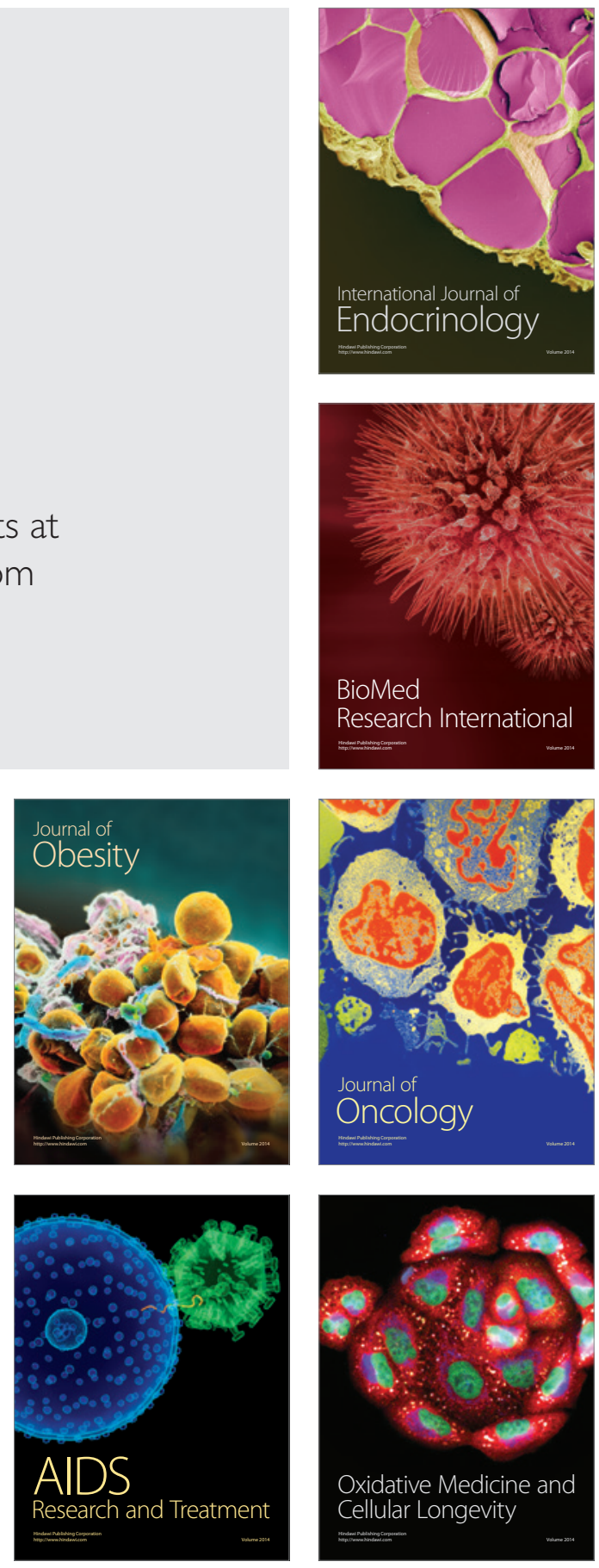